\title{
La valutazione della malnutrizione in emodialisi con il punteggio Malnutrition Inflammation Score (MIS): esperienza infermieristica
}

\author{
Daria Motta ${ }^{1}$, Arianna Ferrero ${ }^{2}$, Chiara Riposio ${ }^{3}$, Simona Ellena ${ }^{4}$, Roberto Boero ${ }^{1}$ \\ ${ }^{1}$ Dirigente Medico, SC Nefrologia e Dialisi, Ospedale Martini, ASL "Città di Torino", Torino \\ ${ }^{2}$ Dietista, SC Nefrologia e Dialisi, Ospedale Martini, ASL "Città di Torino", Torino \\ ${ }^{3}$ Collaboratore Professionale Sanitario Infermiere (CPSI), SC Nefrologia e Dialisi, Ospedale Martini, ASL "Città di Torino", Torino \\ ${ }^{4}$ Collaboratore Professionale Sanitario Esperto Coordinatore (CPSEC), SC Nefrologia e Dialisi, Ospedale Martini, ASL "Città di Torino", Torino
}

\begin{abstract}
Use of malnutrition inflammation score in hemodialysis patients by hemodialysis nurses
Malnutrition inflammation syndrome is common in chronic hemodialysis patients and may correlate with increased morbidity and mortality. A malnutrition inflammation score (MIS) has been proposed as a comprehensive system to assess the presence of this syndrome. We evaluated MIS in a cohort of patients undergoing regular hemodialysis. Furthermore, a dietary questionnaire was administered to patients classified as malnourished according to the MIS score. The MIS score was reassessed after a mean of 22 months in the subgroup of patients still in follow-up. A total of 80 patients on regular hemodialysis ( 45 males and 35 females, mean age $71 \pm 15$ years) were studied; they were classified into 3 groups according to the MIS score: 1) 34 (42.5\%) with good nutrition; 2) 35 (44\%) with mild malnutrition; 3) 11 $(13.5 \%)$ with moderate to severe malnutrition. Malnourished patients were significantly older than others, while the duration of dialysis was similar. Survival during 26 months' follow-up was statistically different between the 3 groups (Cox-Mantel test, $p=0.009$ ) and was $75 \%$ in group $1,49 \%$ in group 2 and $40 \%$ in group 3 . A dietary questionnaire was administered to the 46 patients of groups 2 and 3 . The results showed that in only $9 \%$ of cases dietary intake was poor, while the remaining cases were equally distributed between those with good and median intake; the MIS score was not different between the 3 groups. In 46 patients the MIS score was reassessed and had worsened significantly in patients without malnutrition at baseline; conversely, it significantly improved in those previously found to be malnourished who received dietary counseling. Our study shows that the MIS score may predict patient survival.
\end{abstract}

Keywords: Dietary counseling, Hemodialysis, Inflammation, Malnutrition, MIS score

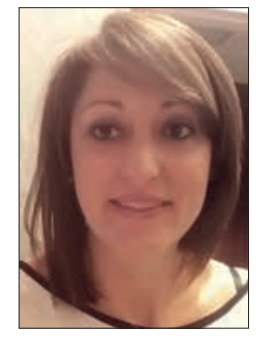

Daria Motta

\section{Introduzione}

La presenza di malnutrizione proteico-calorica è una condizione temibile nei pazienti con insufficienza renale cronica sottoposti a trattamento dialitico. I pazienti con insufficienza renale cronica nei vari stadi della malattia spesso riducono l'apporto proteico-calorico; inoltre, nel

Accepted: July 13, 2017

Published online: September 12, 2017

Indirizzo per la corrispondenza:

Dott.ssa Daria Motta

SC Nefrologia e Dialisi

Ospedale Martini, ASL "Città di Torino"

Via Tofane, 71

10141 Torino

dariamotta@gmail.com
10-30\% dei pazienti in dialisi la malnutrizione si associa alla presenza di infiammazione e allo sviluppo di aterosclerosi e costituisce una delle principali cause di morbilità e di mortalità (1).

Lo screening nutrizionale è un metodo rapido e semplice, somministrabile a tutti i pazienti all'accettazione in ospedale $\mathrm{o}$ in altra istituzione. Kalantar-Zadeh et al (2) hanno proposto il Malnutrition Inflammation Score (MIS), un indice che comprende 7 componenti dello Subjective Global Assessment (SGA) e altri 3 non-SGA: I'indice di massa corporea (BMI), I'albumina sierica e la capacità ferro-legante totale (TIBC). In letteratura, il MIS ha mostrato un'elevata capacità discriminante di malnutrizione ed è risultato altamente predittivo di morbilità e mortalità (2-4). II MIS rappresenta uno strumento di valutazione nutrizionale per i pazienti in emodialisi ma, dal momento che comprende una valutazione soggettiva, richiede un'accurata formazione del personale.

Scopo di questo lavoro è descrivere un'esperienza infermieristica di applicazione del MIS a una coorte di pazienti in 
trattamento emodialitico, per valutarne la fattibilità, il valore predittivo nei confronti della mortalità, nonché le sue eventuali variazioni nel tempo a seguito di un intervento di counseling nutrizionale.

\section{Casistica}

Sono stati studiati 80 degli 87 pazienti in trattamento dialitico extracorporeo presso il Centro Dialisi dell'Ospedale Martini di Torino nel maggio 2014, 45 maschi e 35 femmine, di età media $71 \pm 15$ anni, età mediana 72,5 anni, intervallo interquartile 5,5 anni. I rimanenti 7 pazienti non sono stati studiati per la presenza, al momento dell'indagine, di importante deterioramento cognitivo, neoplasia in stadio terminale o barriere sociali e linguistiche. Trentatré pazienti (41\%) erano trattati con emodialisi con bicarbonato (BHD), 36 (45\%) con emodiafiltrazione on-line (HDF), 7 (9\%) con biofiltrazione senza acetato (AFB), 4 (5\%) con emodiafiltrazione con reinfusione endogena (HFR). L'accesso vascolare era rappresentato da fistola artero-venosa in 47 casi, catetere venoso centrale a permanenza in 33 casi. L'età dialitica media era $88 \pm 97$ mesi, mediana 50 mesi, intervallo interquartile 68 mesi. Le nefropatie di base comprendevano: nefroangiosclerosi $(n=33)$, diabete mellito $(n=12)$, pielonefrite cronica $(n=12)$, nefropatia policistica $(n=8)$, glomerulonefriti $(n=7)$, ipoplasia renale ( $n$ $=2)$, vasculite $(n=1)$, nefropatia non diagnosticata $(n=5)$.

\section{Metodi}

È stato utilizzato il questionario MIS come originariamente proposto da Kalantar-Zadeh et al (2). Gli infermieri del reparto sono stati addestrati all'utilizzo del MIS in occasione di un corso di formazione interno tenuto da dietologi e dietisti esperti. Per tutti i pazienti è stato calcolato il punteggio MIS nel mese di maggio 2014. I pazienti sono stati successivamente osservati fino a 26 mesi. Nel mese di febbraio 2016 il punteggio MIS è stato ricalcolato per protocollo, nei pazienti ancora in osservazione $(n=46)$, al fine di evidenziare eventuali variazioni; gli infermieri che hanno condotto la seconda rilevazione del MIS non erano necessariamente gli stessi che avevano eseguito la precedente. In base al punteggio MIS ottenuto al basale i pazienti sono stati suddivisi in tre gruppi: Gruppo 1 con buona nutrizione (punteggio da 0 a 5); Gruppo 2 con lieve malnutrizione (punteggio da 6 a 10); Gruppo 3 con moderata o severa malnutrizione (punteggio $\geq 11$ ).

Ai pazienti con punteggio MIS basale $>5$ gli infermieri hanno somministrato anche un diario alimentare guidato per valutare la qualità e la quantità dei nutrienti assunti, elaborato da un gruppo di dietologi e dietisti e reperibile nel documento Proposte operative per la ristorazione assistenziale della Regione Piemonte (5). In breve, si tratta di una scheda nella quale viene attribuito un punteggio in base alla porzione consumata abitualmente di ciascun alimento o alla portata nei tre pasti principali e in due eventuali spuntini; il punteggio varia da 0 (nulla) a: 0,25 (1/4); 0,5 (1/2); 0,75 (3/4); 1 (tutta). In base alla somma dei punteggi del diario alimentare $i$ pazienti sono stati suddivisi in tre gruppi: con alimentazione scarsa (punteggio 0-5), media (punteggio 6-9), buona (punteggio 10-12). In tutti i 46 pazienti nei quali è stato ripetuto il punteggio MIS è stato anche rivalutato il diario alimentare; di questi, 21 avevano effettuato anche la valutazione al basale in quanto avevano un punteggio MIS $>5$; è stato eseguito il confronto tra questi due gruppi.

In tutti i casi con punteggio MIS indicativo di uno stato di malnutrizione lieve o moderata/severa è stato effettuato durante il periodo di osservazione un intervento di counseling alimentare da parte del personale infermieristico, attraverso un colloquio di educazione sanitaria e la consegna di opusco$\mathrm{li}$; in 21 pazienti, segnalati a giudizio degli infermieri, vi è stato anche l'intervento della dietista che, sulla base dell'anamnesi alimentare e del confronto con i fabbisogni stimati, ha elaborato e spiegato ai pazienti o ai caregiver schemi alimentari personalizzati che comprendevano eventualmente anche I'impiego di integratori alimentari per os. I pazienti inviati alla dietista non differivano per le diverse variabili basali esaminate da quelli non inviati.

I dati sono stati elaborati con il software StatSoft Statistica. I risultati sono espressi come media e deviazione standard oppure come mediana e intervallo interquartile quando appropriato. La differenza tra i gruppi è stata calcolata con I'analisi della varianza o il test $\mathrm{t}$ di Student per gruppi o dati appaiati. La differenza tra le frequenze è stata analizzata con il test Chi quadro. L'analisi della sopravvivenza è stata effettuata con il test di Cox-Mantel.

\section{Risultati}

II valore medio del punteggio MIS al basale è stato $6,5 \pm$ 3,6 , il valore mediano 6 (intervallo interquartile 5,5). Non sono state riscontrate differenze significative tra maschi e femmine (rispettivamente $7,8 \pm 3,8$ vs $6,6 \pm 2,8$ ). Trentaquattro pazienti $(42,5 \%)$ avevano alla valutazione basale una buona nutrizione, 35 (44\%) una lieve malnutrizione, 11 (13,5\%) una moderata o severa malnutrizione.

Confrontando i tre gruppi (Tab. I) si osserva che i pazienti malnutriti sono significativamente più anziani $(p=0,006)$, mentre l'età dialitica è simile. L'indice di massa corporea è significativamente inferiore soprattutto per i pazienti con malnutrizione moderata o severa $(p=0,02)$. I valori di albuminemia e di transferrina sono significativamente inferiori nei pazienti con malnutrizione $(p=0,0004$ e $p=0,005$ rispettivamente). La frequenza di vasculopatia periferica e di diabete è significativamente superiore nei pazienti con moderata malnutrizione ( $p=0,002$ e $p=0,003$ rispettivamente). La dose di eritropoietina e l'indice di resistenza all'eritropoietina (ERI) sono più elevati nei pazienti con diversi gradi di malnutrizione. La differenza diventa significativa confrontando i pazienti suddivisi in solo due gruppi, senza e con malnutrizio- 
TABELLA I - Caratteristiche anagrafiche e cliniche in base al punteggio MIS al basale

\begin{tabular}{|c|c|c|c|c|}
\hline Punteggio MIS & $\begin{array}{c}0-5 \\
\begin{array}{c}\text { Stato nutrizionale } \\
\text { normale }\end{array}\end{array}$ & $\begin{array}{c}\text { 6-10 } \\
\text { Malnutrizione lieve }\end{array}$ & $\begin{array}{l}\qquad 11 \\
\text { Malnutrizione moderata/ } \\
\text { severa }\end{array}$ & $\mathbf{p}$ \\
\hline Numero casi (\%) & $34(42,5 \%)$ & $35(44 \%)$ & $11(13,5 \%)$ & \\
\hline Età (anni) & $64,9 \pm 18$ & $73,5 \pm 12$ & $79,6 \pm 9$ & 0,006 \\
\hline Punteggio MIS & $3,0 \pm 0,2$ & $8,0 \pm 0,2$ & $12,4 \pm 0,4$ & 0,01 \\
\hline Punteggio diario alimentare basale & Non eseguito & $5,6 \pm 2,2$ & $5,2 \pm 2,3$ & n.s. \\
\hline Età dialitica (mesi) & $89 \pm 99$ & $87 \pm 107$ & $88 \pm 52$ & n.s. \\
\hline BMI $\left(\mathrm{kg} / \mathrm{m}^{2}\right)$ & $24,9 \pm 4,6$ & $24,9 \pm 5,2$ & $20,4 \pm 5,2$ & 0,02 \\
\hline Albumina $(\mathrm{g} / \mathrm{dL})$ & $4,0 \pm 0,3$ & $3,7 \pm 0,3$ & $3,5 \pm 0,3$ & 0,0004 \\
\hline Transferrina (mg/dL) & $213 \pm 43$ & $192 \pm 43$ & $165 \pm 43$ & 0,005 \\
\hline Emoglobina (g/dL) & $10,9 \pm 1$ & $10,3 \pm 1$ & $11,1 \pm 1$ & n.s. \\
\hline Dose di Eritropoietina (U/kg/settimana) & $123 \pm 70$ & $156 \pm 74$ & $158 \pm 73$ & n.s. \\
\hline $\begin{array}{l}\text { Indice di resistenza all'Eritropoietina (U/kg/ } \\
\text { settimana/g/dL) }\end{array}$ & $11,6 \pm 7$ & $15,3 \pm 7$ & $14,7 \pm 7$ & n.s. \\
\hline Vasculopatia periferica & $4(12 \%)$ & $15(43 \%)$ & $1(9 \%)$ & 0,002 \\
\hline Cardiopatia ischemica & $11(32 \%)$ & $20(57 \%)$ & $6(54 \%)$ & n.s. \\
\hline Scompenso cardiaco & $4(12 \%)$ & $1(3 \%)$ & $2(18 \%)$ & n.s. \\
\hline Diabete mellito & $5(15 \%)$ & $15(44 \%)$ & $0(0 \%)$ & 0,003 \\
\hline
\end{tabular}

TABELLA II - Confronto tra pazienti vivi e deceduti al termine del periodo di osservazione

\begin{tabular}{|c|c|c|c|}
\hline & Vivi & Deceduti & $\mathbf{p}$ \\
\hline Numero casi & 48 & 32 & \\
\hline Età (anni) & $65 \pm 15$ & $79 \pm 11$ & 0,001 \\
\hline Età dialitica (mesi) & $104 \pm 117$ & $64 \pm 46$ & n.s. \\
\hline $\mathrm{BMI}\left(\mathrm{kg} / \mathrm{m}^{2}\right)$ & $25 \pm 5$ & $23 \pm 4$ & n.s. \\
\hline Albumina $(\mathrm{g} / \mathrm{dL})$ & $3,9 \pm 0,4$ & $3,7 \pm 0,4$ & 0,02 \\
\hline Transferrina (mg/dL) & $202 \pm 49$ & $191 \pm 38$ & n.s. \\
\hline Emoglobina (g/dL) & $10,8 \pm 1$ & $10,5 \pm 1$ & n.s. \\
\hline Dose di Eritropoietina (U/kg/settimana) & $128 \pm 77$ & $164 \pm 63$ & 0,04 \\
\hline Indice di resistenza all'Eritropoietina (U/kg/settimana/g/dL) & $12,2 \pm 7$ & $15,8 \pm 6$ & 0,037 \\
\hline Punteggio MIS basale & $5,6 \pm 3,6$ & $7,8 \pm 3,2$ & 0,017 \\
\hline Punteggio diario alimentare basale & $5,6 \pm 2,0$ & $5,5 \pm 2,4$ & n.s. \\
\hline Diabete & $12(25 \%)$ & $8(25 \%)$ & n.s. \\
\hline Scompenso cardiaco & $1(2 \%)$ & $5(16 \%)$ & 0,03 \\
\hline Cardiopatia ischemica & 19 (40\%) & $18(56 \%)$ & n.s. \\
\hline Vasculopatia periferica & $8(17 \%)$ & $11(34 \%)$ & 0,06 \\
\hline
\end{tabular}

ne (rispettivamente dose di eritropoietina $123 \pm 63$ vs $156 \pm$ $77 \mathrm{U} / \mathrm{kg} /$ settimana, $\mathrm{p}=0,05 ; \mathrm{ERI} 11,6 \pm 6$ vs 15,1 $\pm 7 \mathrm{U} / \mathrm{kg} /$ settimana/g/dL, $p=0,036)$.

Nel corso del periodo di osservazione 35 pazienti sono usciti dallo studio: 32 per decesso, 2 per trasferimento ad altro Centro, 1 per trapianto renale. II confronto tra i deceduti e i sopravvissuti (Tab. II) evidenzia che i primi avevano un'età anagrafica e un punteggio MIS significativamente superiori ( $p=0,001$ e $p=0,017$ rispettivamente), mentre l'età dialitica e l'indice di massa corporea non erano diversi in maniera rilevante. I valori di albuminemia erano significativamente inferiori nei deceduti $(p=0,02)$. Lo scompenso cardiaco e la vasculopatia periferica erano più frequenti nei pazienti deceduti ( $p=0,03$ e $p=0,06$ rispettivamente). Anche la dose di eritropoietina e l'ERI sono risultati statisticamente più elevati nei pazienti deceduti ( $p=0,04$ e $p=0,037$ rispettivamente).

L'analisi della sopravvivenza ha evidenziato una differenza statisticamente significativa $(p=0,009)$ nei tre gruppi in base al valore del punteggio MIS (Fig. 1); la sopravvivenza attuariale è stata del 75\% nel Gruppo 1, del 49\% nel Gruppo 2 e del 40\% nel Gruppo 3.

Ripetendo l'analisi di sopravvivenza nei pazienti suddivisi in due gruppi in base all'età anagrafica inferiore o superiore alla mediana (72,5 anni), si osserva che nei pazienti con età 


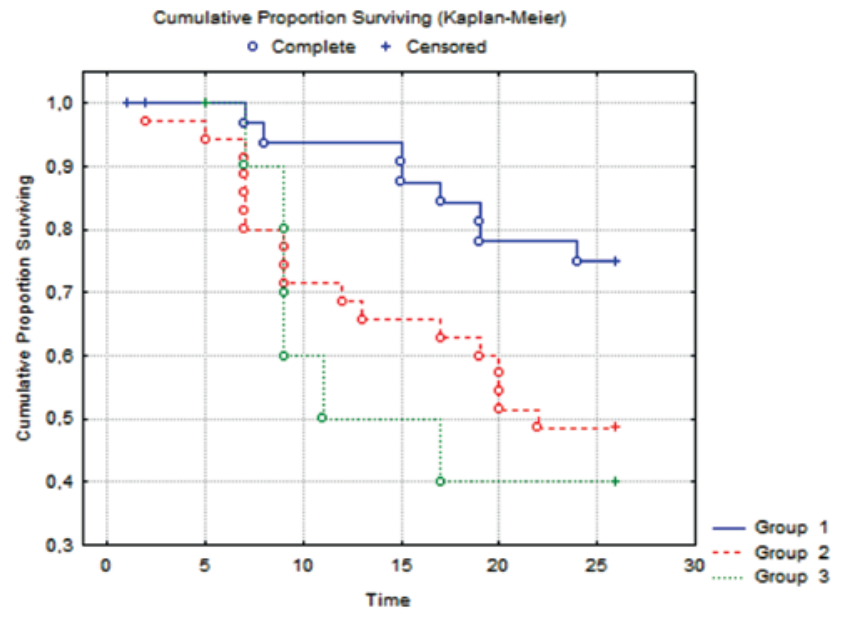

Fig. 1 - Curve di sopravvivenza nei pazienti suddivisi in base al valore MIS basale. Gruppo 1 con buona nutrizione (punteggio da 0 a 5); Gruppo 2 con lieve malnutrizione (punteggio da 6 a 10); Gruppo 3 con moderata o severa malnutrizione (punteggio $\geq 11)(p=0,009)$.

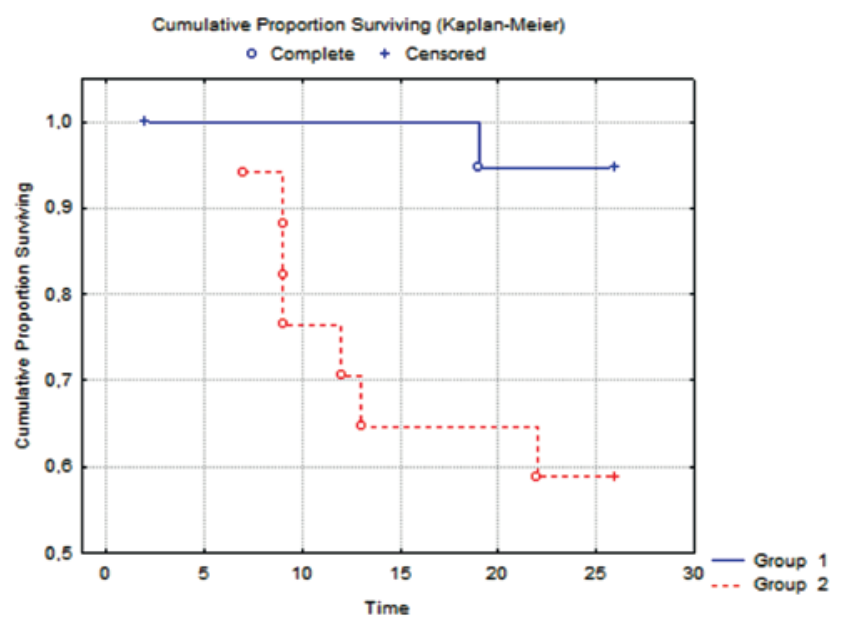

Fig. 2 - Curve di sopravvivenza nei pazienti con età anagrafica $<72,5$ anni, suddivisi in base al valore MIS basale. Gruppo 1 con buona nutrizione (punteggio da 0 a 5); Gruppo 2 con malnutrizione (punteggio $>5)(p=0,01)$.

inferiore il punteggio MIS influenza in modo significativo la sopravvivenza (sopravvivenza attuariale del 95\% nei pazienti con MIS 0-5 vs $59 \%$ nei pazienti con MIS >5, $p=0,01$ ) (Fig. 2), mentre nei pazienti con età anagrafica superiore la sopravvivenza attuariale era del $39 \%$ nei pazienti con MIS 0-5 vs 36\% nei pazienti con MIS >5, differenza non statisticamente significativa (Fig. 3).

Nei 46 pazienti ancora in osservazione dopo 22 mesi è stato rivalutato il punteggio MIS (Tab. III). Rispetto ai pazienti che erano usciti dallo studio i pazienti nei quali è stato rivalutato il punteggio MIS avevano un'età anagrafica significativamente inferiore $(64,8 \pm 15$ vs $78.6 \pm 11$ anni, $p=0,0002)$, un BMI più elevato $\left(25,5 \pm 5,6\right.$ vs $\left.22,7 \pm 3,9 \mathrm{~kg} / \mathrm{m}^{2}, \mathrm{p}=0,016\right)$

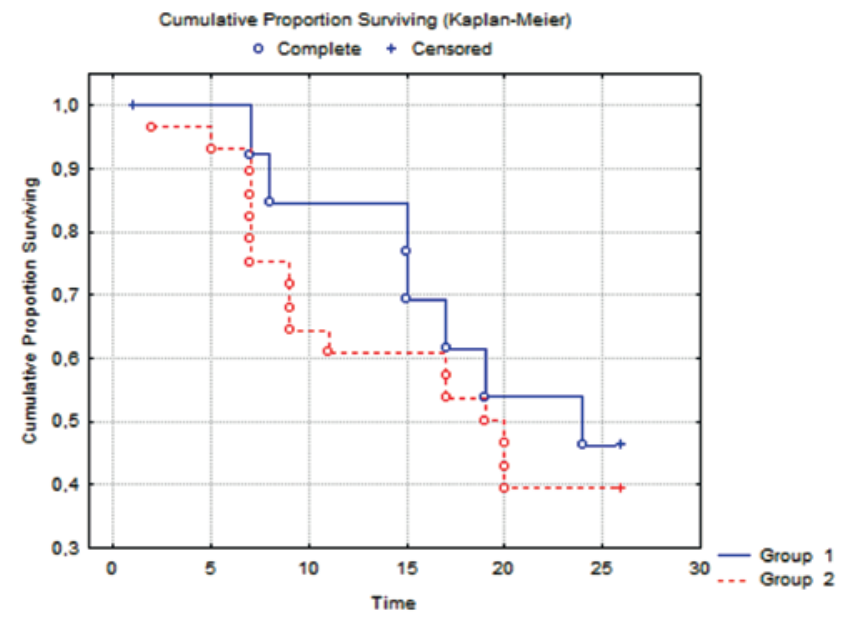

Fig. 3 - Curve di sopravvivenza nei pazienti con età anagrafica $>72,5$ anni, suddivisi in base al valore MIS basale. Gruppo 1 con buona nutrizione (punteggio da 0 a 5); Gruppo 2 con malnutrizione (punteggio $>5)(p=n . s$.$) .$

e un punteggio MIS basale inferiore $(5,5 \pm 3,5$ vs $7,8 \pm 3,2$, $p=0,004)$. Nella casistica globale il punteggio è rimasto sostanzialmente invariato. Suddividendo i pazienti in base alla presenza o assenza di malnutrizione alla valutazione basale si osserva che mentre in questi ultimi il punteggio peggiora significativamente $(p=0,03)$, nei pazienti con malnutrizione il punteggio migliora, in maniera significativa solo nel gruppo con malnutrizione di grado lieve $(p=0,049)$. Nei 46 pazienti con punteggio MIS al basale $>5$ è stato somministrato anche il diario alimentare come descritto precedentemente. I risultati sono presentati nella Tabella IV. I dati evidenziano che solo nel 9\% dei pazienti l'alimentazione era scarsa, mentre i restanti casi si dividevano equamente tra pazienti con alimentazione buona e media. In 21 pazienti è stato confrontato il punteggio del diario alimentare basale e dopo un intervallo di 22 mesi. I risultati evidenziano un incremento significativo del punteggio da $5,5 \pm 2$ a $6,9 \pm 3(p=0,03)$.

In tutti i casi con punteggio MIS basale indicativo di uno stato di malnutrizione lieve o moderata/severa $(n=46)$ è stato effettuato durante il periodo di osservazione un intervento di counseling alimentare da parte del personale infermieristico; in 21 di essi vi è stato anche l'intervento della dietista, con attività di consulenza specialistica $(n=18)$ e con prescrizione di integratori alimentari $(n=3)$. Il punteggio del diario alimenta-

TABELLA III - Variazioni del punteggio MIS durante il periodo di osservazione in base ai valori basali

\begin{tabular}{lccc}
\hline & $\begin{array}{c}\text { Punteggio MIS } \\
\text { basale }\end{array}$ & $\begin{array}{c}\text { Punteggio MIS } \\
\text { al follow-up }\end{array}$ & p \\
\hline Casistica globale & $5,5 \pm 3,6$ & $5,3 \pm 3,2$ & n.s. \\
MIS 0-5 al basale & $2,64 \pm 1,2$ & $3,8 \pm 2,6$ & 0,03 \\
MIS 6-10 al basale & $8,05 \pm 1,3$ & $6,6 \pm 2,8$ & 0,049 \\
MIS $\geq 11$ al basale & $12,5 \pm 0,6$ & $9,5 \pm 3,1$ & n.s. \\
\hline
\end{tabular}


TABELLA IV - Classificazione in base ai risultati del diario alimentare

\begin{tabular}{|c|c|c|c|c|}
\hline & Alimentazione buona & Alimentazione media & Alimentazione scarsa & p \\
\hline Punteggio diario alimentare basale & $3,8 \pm 0,2$ & $6,4 \pm 0,2$ & $10,1 \pm 0,5$ & 0,0001 \\
\hline Età anagrafica (anni) & $74 \pm 2$ & $76 \pm 2$ & $71 \pm 6$ & n.s. \\
\hline Età dialitica (mesi) & $89 \pm 20$ & $64 \pm 20$ & $202 \pm 45$ & 0,028 \\
\hline
\end{tabular}

re è migliorato maggiormente nei pazienti che hanno ricevuto I'intervento della dietista rispetto a quelli che non lo hanno avuto anche se in maniera non statisticamente significativa: differenza, espressa come mediana e intervallo interquartile $1,37(4,7)$ vs $0,52(2,45)$.

\section{Discussione}

I nostri dati evidenziano che circa il $58 \%$ dei pazienti in emodialisi presenta uno stato di malnutrizione/infiammazione da lieve a moderato-severo. Tale dato è in linea con quanto riscontrato da altri autori $(2,6,7)$, nonostante non sia rappresentativo dell'intera popolazione dei pazienti emodializzati nella nostra area, in quanto la presente casistica comprende solo i pazienti del Centro ospedaliero e non i 40 pazienti trattati presso il Centro ad Assistenza limitata, solitamente in migliori condizioni cliniche generali. II punteggio MIS è risultato correlato all'età anagrafica dei pazienti ma non all'età dialitica, come già osservato da altri autori (2).

Il punteggio MIS è fortemente predittivo della prognosi dei pazienti, confermando precedenti osservazioni (7-12); a questo proposito occorre tenere presente che il punteggio MIS incrementa con l'età anagrafica e che quest'ultima influenza in maniera indipendente la mortalità. Per questo motivo l'analisi della sopravvivenza è stata ripetuta considerando separatamente i pazienti con età inferiore o superiore alla mediana (72,5 anni). Soltanto nei pazienti più giovani si conferma il significato prognostico del punteggio MIS, mentre nei pazienti più anziani l'età assume un ruolo dominante in grado di oscurare il valore predittivo del punteggio MIS.

Nel nostro studio, nei pazienti con valori di MIS al basale indicativi di uno stato di malnutrizione-infiammazione, i dati sono stati corroborati con l'analisi dell'alimentazione ottenuta tramite la compilazione di un questionario alimentare guidato, utilizzato nell'ambito della ristorazione assistenziale. In questo modo si è cercato di discriminare se il peggiore stato nutrizionale fosse associato anche a una minore ingestione di nutrienti. I risultati evidenziano che una scarsa alimentazione era presente solo nel $9 \%$ dei casi e che non vi erano correlazioni tra il punteggio MIS e il punteggio del diario alimentare. Questa osservazione è in accordo con precedenti analisi (6) e suggerisce che il ridotto introito calorico/proteico sembra avere un peso inferiore rispetto agli altri determinanti del punteggio MIS e che quest'ultimo risulta quindi maggiormente influenzato dalla presenza di infiammazione sistemica. A questo proposito è interessante sottolineare che anche i nostri dati evidenziano una correlazione tra valori elevati di punteggio MIS e resistenza all'eritropoietina, possibile espressione di uno stato infiammatorio cronico $(2,13)$. Una scarsa alimentazione sembra essere presente soprattutto nei pazienti con età dialitica molto elevata, infatti i 4 pazienti con alimentazione scarsa avevano un'anzianità dialitica nettamente e significativamente superiore a quelli con alimentazione buona o media.

II questionario MIS, ripetuto dopo un periodo di 22 mesi, ha mostrato che nei pazienti con buona nutrizione al basale il punteggio tende a peggiorare, mentre migliora significativamente nei pazienti con peggiore stato di malnutrizione-infiammazione. Complessivamente questi dati vanno interpretati con cautela, in quanto in parte il punteggio MIS è di natura soggettiva e quindi può presentare un certo grado di variabilità tra osservatori diversi (14). Il peggioramento del MIS con il tempo è in parte inevitabile in quanto l'età dialitica contribuisce a determinare il punteggio, tuttavia il dato sottolinea la necessità di monitorare strettamente anche i pazienti con condizioni migliori che possono peggiorare in un follow-up relativamente breve. A questo proposito è particolarmente rilevante il miglioramento riscontrato nel sottogruppo dei pazienti con valori patologici alla valutazione basale; in questi pazienti è stato effettuato un intervento di counseling da parte del personale infermieristico e in alcuni di essi anche da parte della dietista, con prescrizione di schemi nutrizionali personalizzati e in alcuni casi con l'utilizzo di integratori alimentari a domicilio. La natura osservazionale di questo studio non consente di stabilire con certezza se il miglioramento sia da attribuire all'intervento effettuato sui pazienti, come recentemente suggerito da alcuni autori $(15,16)$ in quanto non si dispone di un gruppo di controllo; tuttavia l'osservazione, nonostante sia da interpretare con cautela, è interessante perché potrebbe indicare la possibilità di interferire positivamente con lo stato di malnutrizione. È importante sottolineare che i pazienti che hanno ricevuto un counseling da parte di una dietista specializzata in nutrizione dei pazienti nefropatici hanno avuto, al follow-up, un incremento maggiore del punteggio del questionario alimentare, ancorché non statisticamente significativo, rispetto a quelli che non lo avevano avuto. È nostra opinione che, mentre in tutti i pazienti gli infermieri debbano svolgere un'azione di counseling 
alimentare, nei casi con malnutrizione è auspicabile sempre I'intervento specialistico di un dietista.

In conclusione lo studio dimostra che il punteggio MIS è uno strumento che può aiutare a stratificare la prognosi dei pazienti e potenzialmente a identificare i soggetti nei quali è indicato un intervento per migliorare lo stato di nutrizione e infiammazione.

\section{Disclosures}

Financial support: No financial support was received for this submission. Conflict of interest: The authors have no conflict of interest.

Il comitato Etico ha ritenuto l'autorizzazione non necessaria.

\section{Bibliografia}

1. Ikizler TA, Cano NJ, Franch H, et al. International Society of Renal Nutrition and Metabolism. Prevention and treatment of protein energy wasting in chronic kidney disease patients: a consensus statement by the International Society of Renal Nutrition and Metabolism. Kidney Int. 2013;84(6): 1096-1107.

2. Kalantar-Zadeh K, Kopple JD, Block G, Humphreys MH. A malnutrition-inflammation score is correlated with morbidity and mortality in maintenance hemodialysis patients. Am J Kidney Dis. 2001;38(6):1251-63.

3. Bonanni A, Mannucci I, Verzola D, et al. Protein-energy wasting and morbidity in chronic kidney disease. Int J Environ Res PublicHealth. 2011;8(5):1631-54.

4. Rambod M, Bross R, Zitterkoph J, et al. Association of Malnutrition-Inflammation Score with quality of life and mortality in hemodialysis patients: a 5-year prospective cohort study. Am J Kidney Dis. 2009;53(2):298-309.

5. Amerio ML, Pezzana A. Proposte operative per la ristorazione assistenziale, Regione Piemonte, Direzione Sanità Pubblica, 2007. http://www.regione.piemonte.it/sanita/cms2/ documentazione/category/152-rete-dietistica-e-nutrizioneclinica?download = 3296: proposte-operative-per-la-ristorazione-assistenziale-2007

6. Chen J, Peng H, Xiao L, et al. Inflammation but not dietary macronutrients insufficiency associated with the malnutritioninflammation score in hemodialysis population. PLoS One. 2013;8(12):e83233.

7. Borgio C, Forneris G, Pezzana A, Roccatello D. L'utilizzo del test di screening nutrizionale Malnutrition Inflammation Score (MIS) in dialisi. G Tec Nefrol Dial. 2015;27(4):226-31.

8. He T, An X, Mao HP, et al. Malnutrition-inflammation score predicts long-term mortality in Chinese PD patients. Clin Nephrol. 2013;79(6):477-83.

9. Pisetkul C, Chanchairujira K, Chotipanvittayakul N, OngAjyooth L, Chanchairujira T. Malnutrition-inflammation score associated with atherosclerosis, inflammation and short-term outcome in hemodialysis patients. J Med Assoc Thai. 2010;93 (Suppl 1):S147-56.

10. Kara E, Sahutoglu T, Ahbap E, et al. The predictive value of malnutrition-inflammation score on 1-year mortality in Turkish maintenance hemodialysis patients. Clin Nephrol. 2016;86(2):94-9.

11. Perez Vogt B, Costa Teixeira Caramori J. Are nutritional composed scoring systems and protein-energy wasting score associated with mortality in maintenance hemodialysis patients? J Ren Nutr. 2016;26(3):183-9.

12. Santin FG, Bigogno FG, Dias Rodrigues JC, Cuppari L, Avesani $\mathrm{CM}$. Concurrent and predictive validity of composite methods to assess nutritional status in older adults on hemodialysis. $J$ Ren Nutr. 2016;26(1):18-25.

13. Yamada K, Furuya R, Takita $T$, et al. Simplified nutritional screening tools for patients on maintenance hemodialysis. Am J Clin Nutr. 2008;87:106-13.

14. Beberashvili I, Azar A, Sinuani I, et al. Comparison analysis of nutritional scores for serial monitoring of nutritional status in hemodialysis patients. Clin J Am Soc Nephrol. 2013;8(3): 443-51.

15. Sohrabi Z, Eftekhari MH, Eskandari MH, Rezaianzadeh A, Sagheb MM. Intradialytic oral protein supplementation and nutritional and inflammation outcomes in hemodialysis: A Randomized Controlled Trial. Am J Kidney Dis. 2016;68(1): 122-30.

16. Roy LG, Shetty MS, Urooj A. Effect of nutritional intervention on malnutrition indicators in patients on haemodialysis. J Ren Care. 2013;39(1):39-46. 